MITSUBISHI ELECTRIC RESEARCH LABORATORIES

http://www.merl.com

\title{
Network-Coded Interference Alignment in K-Pair Bidirectional Relaying Channels
}

\author{
Koike-Akino, T.; Pun, M-O.; Orlik, P.
}

TR2011-031 June 2011

\begin{abstract}
AbstractIn this paper, we propose a distributed interference alignment which employs physicallayer network coding and superposition coding for successive-canceling multiuser detection (MUD) receivers in K-pair bidirectional relaying networks. The proposed scheme enables the transmitter to align only partial interference while strong interference is canceled by MUD, and the transmitter can have more degrees of freedom in controlling the filter designs. Simulation results demonstrate that our proposed scheme significantly improves sum-rate performance in multiuser bidirectional relaying systems.
\end{abstract}

IEEE International Conference on Communications (ICC)

This work may not be copied or reproduced in whole or in part for any commercial purpose. Permission to copy in whole or in part without payment of fee is granted for nonprofit educational and research purposes provided that all such whole or partial copies include the following: a notice that such copying is by permission of Mitsubishi Electric Research Laboratories, Inc.; an acknowledgment of the authors and individual contributions to the work; and all applicable portions of the copyright notice. Copying, reproduction, or republishing for any other purpose shall require a license with payment of fee to Mitsubishi Electric Research Laboratories, Inc. All rights reserved.

Copyright (C) Mitsubishi Electric Research Laboratories, Inc., 2011

201 Broadway, Cambridge, Massachusetts 02139 



\title{
Network-Coded Interference Alignment in $K$-Pair Bidirectional Relaying Channels
}

\author{
Toshiaki Koike-Akino, Man-On Pun, and Philip Orlik, \\ Mitsubishi Electric Research Laboratories (MERL), 201 Broadway, Cambridge, MA 02139, U.S.A. \\ Email: \{koike, porlik\}@merl.com
}

\begin{abstract}
In this paper, we propose a distributed interference alignment which employs physical-layer network coding and superposition coding for successive-cancelling multiuser detection (MUD) receivers in $K$-pair bidirectional relaying networks. The proposed scheme enables the transmitter to align only partial interference while strong interference is cancelled by MUD, and the transmitter can have more degrees of freedom in controlling the filter designs. Simulation results demonstrate that our proposed scheme significantly improves sum-rate performance in multiuser bidirectional relaying systems.
\end{abstract}

\section{INTRODUCTION}

Radio communications are often exposed by possible interference due to simultaneous transmitters, in particular for distributed wireless networks. The capacity region of such interference channels, even in Gaussian cases, has been an open question for over three decades [1]. One known result is about a two-user strong interference case in which each receiver has a better reception of the unintended signals than the intended data [1-3]. Unfortunately, no results on the general case are available in the literature. Instead of analyzing the exact capacity region, there have emerged much interests in studying an asymptotic network capacity such as achievable degrees of freedom (or, the slope of capacity curves) in high signal-to-noise power ratio (SNR) regimes. A transmission technique termed interference alignment [4-9] was introduced to exploit high degrees of freedom in multiuser interference channels. It has been shown that this technique can realize interference-free channels using almost a half of available degrees of freedom in high SNR regimes. The key idea behind interference alignment is to confine interference into a lowerdimensional subspace at each receiver such that interferencefree subspace is reserved for desired signals.

In the conventional interference alignment, distributed users require global channel state information (CSI) for interference suppressions. An alternative method of distributed interference alignment was proposed in [7], where each user requires only the local CSI. This technique makes effective use of a channel reciprocity for iterative filtering optimizations. In this paper, we introduce the same methodology to network-coded relaying systems. A network-coded interference alignment was first investigated by Lee et al. in [11] for the system model, where there are three users and one relaying node. The achievable degrees of freedom was analyzed for such a system. We focus on a different system model, in which there are $K$-pair users as well as relaying nodes for each pair (in total $3 K$ nodes).
All the $2 K$ terminal users simultaneously transmit own data towards the corresponding relaying node in the uplink step, and the relaying nodes broadcast a network-coded signal to all the terminals at the same time in the downlink step.

To further enhance the network performance, we consider a distributed interference alignment in conjunction with multiuser detection (MUD) approach, in which the transmitter aligns only partial interference and the MUD receiver removes intentionally misaligned interference. With MUD, it is expected that the network capacity can be further improved because we have more degrees of freedom for filter optimizations. For the use of MUD, we introduce hyper-layered superposition coding which multiplexes a larger number of low-rate codewords than the maximum degrees of freedom in order for some receivers to successfully cancel them by successive cancelling decoders. This is an extended scheme from the one introduced by Han and Kobayashi in [3]. Simulation results confirm that the proposed strong-leakage interference alignment offers significant performance improvement over the conventional interference alignment.

Notations: We describe matrices and vectors by bold-face italic letters in capital cases and lower cases, respectively. Let $\boldsymbol{X} \in \mathbb{C}^{m \times n}$ be a complex-valued $(m \times n)$-dimensional matrix, where $\mathbb{C}$ denotes the complex field. The notations $\boldsymbol{X}^{*}, \boldsymbol{X}^{\mathrm{T}}, \boldsymbol{X}^{\dagger}, \boldsymbol{X}^{-1}, \operatorname{tr}[\boldsymbol{X}],|\boldsymbol{X}|$, and $\|\boldsymbol{X}\|$ represent the complex conjugate, the transpose, the Hermite transpose, the inverse, the trace, the determinant, and the Frobenius norm of $\boldsymbol{X}$, respectively. The set of real numbers is denoted by $\mathbb{R}$. The $k$-th canonical orthonormal basis vector is written as $\boldsymbol{e}_{k}$, and the $m$-dimensional identity matrix is denoted by $\boldsymbol{I}_{m}$. We define $\mathbb{N}_{K} \triangleq\{1,2, \ldots, K\}$ as a positive integer ring. The expectation operator is written by $\mathbb{E}[\cdot]$.

\section{II. $K$-PAir Bidirectional RELAYing Channels}

\section{A. System Description}

We consider $K$ pairs of communicating terminal users as well as intermediate relaying nodes, as depicted in Fig. 1. For simplicity, we assume all nodes are equipped with $N$ antennas. The $k$-th terminal user (for each pair index $k \in \mathbb{N}_{K}$ ) wishes to exchange data with its partner ( $k^{\prime}$-th user) by a help of the $k$-th relaying node. We make a reasonable assumption that the relaying nodes have larger antenna gains and higher power to forward data (like a base station in cellular networks), and direct links between terminal users are not available for data communications due to a large path loss. All the $2 K$ terminal 


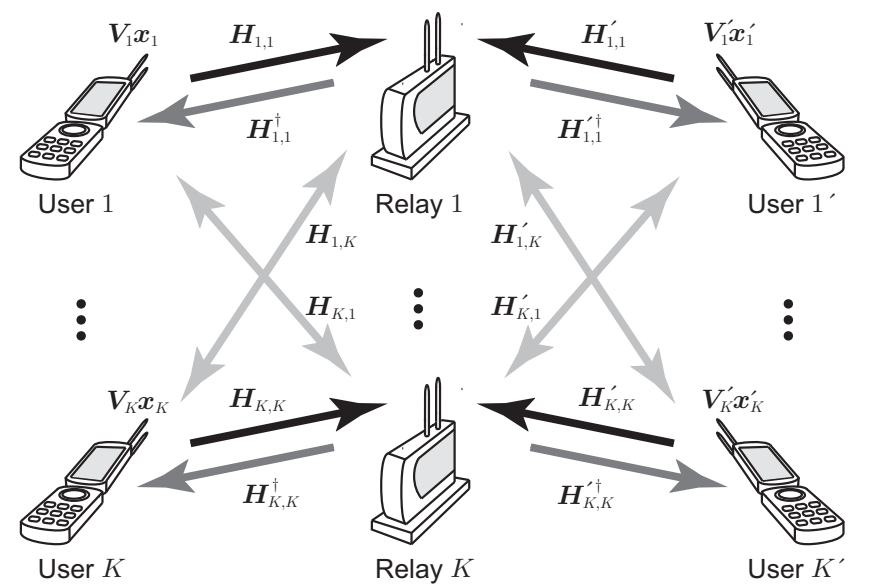

Fig. 1. $K$-pair bidirectional relaying networks for uplink channels $\boldsymbol{H}_{j, i}$ and downlink channels $\boldsymbol{H}_{j, i}^{\dagger}$ with precoding matrices $\boldsymbol{V}_{i}$.

users simultaneously transmit own data towards the relaying nodes in the first uplink step, and all the relaying nodes then broadcast the combined data towards all the users by means of analog network coding in the next downlink step.

\section{B. Bidirectional Relaying Channels}

1) Uplink Step: Let $\boldsymbol{x}_{i} \in \mathbb{C}^{D_{i} \times 1}$ and $\boldsymbol{x}_{i}^{\prime} \in \mathbb{C}^{D_{i}^{\prime} \times 1}$ denote the transmitting data from the $i$-th terminal user and its partner for any $i \in \mathbb{N}_{K}$, where $D_{i}$ and $D_{i}^{\prime}$ are the degrees of freedom for multiplexing data. Multiplexed data $\boldsymbol{x}_{i}$ and $\boldsymbol{x}_{i}^{\prime}$ are transmitted through individual precoding filters, $\boldsymbol{V}_{i} \in \mathbb{C}^{N \times D_{i}}$ and $\boldsymbol{V}_{i}^{\prime} \in \mathbb{C}^{N \times D_{i}^{\prime}}$. In the uplink step, all the $2 K$ terminal users simultaneously transmit those data towards the corresponding relay node. The $j$-th relay (for any $j \in \mathbb{N}_{K}$ ) in turn receives

$$
\boldsymbol{y}_{j}=\sum_{i \in \mathbb{N}_{K}}\left(\boldsymbol{H}_{j, i} \boldsymbol{V}_{i} \boldsymbol{x}_{i}+\boldsymbol{H}_{j, i}^{\prime} \boldsymbol{V}_{i}^{\prime} \boldsymbol{x}_{i}^{\prime}\right)+\boldsymbol{z}_{j},
$$

where $\boldsymbol{y}_{j} \in \mathbb{C}^{N \times 1}, \boldsymbol{H}_{j, i} \in \mathbb{C}^{N \times N}, \boldsymbol{H}_{j, i}^{\prime} \in \mathbb{C}^{N \times N}$, and $\boldsymbol{z}_{j} \in$ $\mathbb{C}^{N \times 1}$ are the received signal, the channel matrix from the $i$-th user to the $j$-th relay, the channel matrix from the $i^{\prime}$-th user to the $j$-th relay, and the additive white Gaussian noise, respectively. Here, we assume $\mathbb{E}\left[\boldsymbol{x}_{i} \boldsymbol{x}_{i}^{\dagger}\right]=\boldsymbol{I}_{D_{i}}, \mathbb{E}\left[\boldsymbol{x}_{i}^{\prime} \boldsymbol{x}_{i}^{\prime \dagger}\right]=$ $\boldsymbol{I}_{D_{i}^{\prime}}, \mathbb{E}\left[\boldsymbol{z}_{j} \boldsymbol{z}_{j}^{\dagger}\right]=\sigma^{2} \boldsymbol{I}_{N}, \operatorname{tr}\left[\boldsymbol{V}_{i} \boldsymbol{V}_{i}^{\dagger}\right]=E$, and $\operatorname{tr}\left[\boldsymbol{V}_{i}^{\prime} \boldsymbol{V}_{i}^{\prime \dagger}\right]=E$, where $\sigma^{2}$ is the noise variance and $E$ is the transmission power per block.

2) Forwarding Filter: The $j$-th relaying node (for $j \in \mathbb{N}_{K}$ ) broadcasts the received signal $\boldsymbol{y}_{j}$ through a forwarding filter $\boldsymbol{P}_{j} \in \mathbb{C}^{N \times N}$, as $\boldsymbol{P}_{j} \boldsymbol{y}_{j}$. This relaying method is called filterand-forward scheme (or simply, amplify-and-forward scheme) in the context of analog network coding at the physical layer. We put a power constraint for the forwarding filter $\boldsymbol{P}_{j}$ at the $j$-th relay as

$$
\begin{aligned}
& \mathbb{E}\left[\left\|\boldsymbol{P}_{j} \boldsymbol{y}_{j}\right\|^{2}\right]=\operatorname{tr}\left[\boldsymbol { P } _ { j } ^ { \dagger } \boldsymbol { P } _ { j } \left(\sigma^{2} \boldsymbol{I}_{N}+\right.\right. \\
& \left.\left.\sum_{i \in \mathbb{N}_{K}}\left(\boldsymbol{H}_{j, i} \boldsymbol{V}_{i} \boldsymbol{V}_{i}^{\dagger} \boldsymbol{H}_{j, i}^{\dagger}+\boldsymbol{H}_{j, i}^{\prime} \boldsymbol{V}_{i}^{\prime} \boldsymbol{V}_{j}^{\prime \dagger} \boldsymbol{H}_{j, i}^{\prime \dagger}\right)\right)\right]=2 E,
\end{aligned}
$$

where $2 E$ is used just for a fair comparison with the conventional one-way relaying system. In addition, it is well-known that two times higher power is asymptotically optimal in the achievable rate for amplify-and-forward schemes.

3) Downlink Step: We suppose that the downlink channel is reciprocal to the uplink cannel, more specifically, the channel from the $j$-th relay to the $k$-th terminal and that from the $j$-th relay to the $k^{\prime}$-th terminal are given as $\boldsymbol{H}_{j, k}^{\dagger}$ and $\boldsymbol{H}_{j, k}^{\prime \dagger}$, respectively. While all the relaying nodes broadcast the network-coded data at the same time, the $k$-th terminal user (for any $k \in \mathbb{N}_{K}$ ) receives

$$
\begin{aligned}
\boldsymbol{r}_{k}= & \sum_{j \in \mathbb{N}_{K}} \boldsymbol{H}_{j, k}^{\dagger} \boldsymbol{P}_{j} \boldsymbol{y}_{j}+\boldsymbol{w}_{k} \\
= & \sum_{j \in \mathbb{N}_{K}} \sum_{i \in \mathbb{N}_{K}} \boldsymbol{H}_{j, k}^{\dagger} \boldsymbol{P}_{j}\left(\boldsymbol{H}_{j, i} \boldsymbol{V}_{i} \boldsymbol{x}_{i}+\boldsymbol{H}_{j, i}^{\prime} \boldsymbol{V}_{i}^{\prime} \boldsymbol{x}_{i}^{\prime}\right) \\
& +\sum_{j \in \mathbb{N}_{K}} \boldsymbol{H}_{j, k}^{\dagger} \boldsymbol{P}_{j} \boldsymbol{z}_{j}+\boldsymbol{w}_{k} \\
\equiv & \sum_{i \in \mathbb{N}_{K}}\left(\boldsymbol{\mathcal { G }}_{k, i} \boldsymbol{V}_{i} \boldsymbol{x}_{i}+\boldsymbol{H}_{k, i} \boldsymbol{V}_{i}^{\prime} \boldsymbol{x}_{i}^{\prime}\right)+\boldsymbol{\xi}_{k},
\end{aligned}
$$

where $\boldsymbol{r}_{k} \in \mathbb{C}^{N \times 1}$ is the received signal and $\boldsymbol{w}_{k} \in \mathbb{C}^{N \times 1}$ is the additive Gaussian noise at the $k$-th terminal user in the downlink step. The matrices $\mathcal{G}_{k, i} \triangleq \sum_{j \in \mathbb{N}_{K}} \boldsymbol{H}_{j, k}^{\dagger} \boldsymbol{P}_{j} \boldsymbol{H}_{j, i}$ and $\boldsymbol{\mathcal { H }}_{k, i} \triangleq \sum_{j \in \mathbb{N}_{K}} \boldsymbol{H}_{j, k}^{\dagger} \boldsymbol{P}_{j} \boldsymbol{H}_{j, i}^{\prime}$ denote the effective channel response from the user $i$ to the user $k$ and that from the $i$ th user's partner (or, user $i^{\prime}$ ) to the user $k$, respectively. The effective noise $\boldsymbol{\xi}_{k} \triangleq \sum_{j \in \mathbb{N}_{K}} \boldsymbol{H}_{j, k}^{\dagger} \boldsymbol{P}_{j} \boldsymbol{z}_{j}+\boldsymbol{w}_{k}$ includes the undesired noise forwarded from the relaying nodes.

Likewise, the $k$-th partner (or, $k^{\prime}$-th terminal user) receives

$$
\boldsymbol{r}_{k}^{\prime}=\sum_{i \in \mathbb{N}_{K}}\left(\mathcal{G}_{k, i}^{\prime} \boldsymbol{V}_{i}^{\prime} \boldsymbol{x}_{i}^{\prime}+\mathcal{H}_{k, i}^{\prime} \boldsymbol{V}_{i} \boldsymbol{x}_{i}\right)+\boldsymbol{\xi}_{k}^{\prime},
$$

where $\boldsymbol{r}_{k}^{\prime} \in \mathbb{C}^{N \times 1}, \mathcal{G}_{k, i}^{\prime} \triangleq \sum_{j \in \mathbb{N}_{K}} \boldsymbol{H}_{j, k}^{\prime \dagger} \boldsymbol{P}_{j} \boldsymbol{H}_{j, i}^{\prime}, \boldsymbol{\mathcal { H }}_{k, i}^{\prime} \triangleq$ $\sum_{j \in \mathbb{N}_{K}} \boldsymbol{H}_{j, k}^{\prime \dagger} \boldsymbol{P}_{j} \boldsymbol{H}_{j, i}, \boldsymbol{\xi}_{k}^{\prime} \triangleq \sum_{j \in \mathbb{N}_{K}} \boldsymbol{H}_{j, k}^{\prime \dagger} \boldsymbol{P}_{j} \boldsymbol{z}_{j}+\boldsymbol{w}_{k}^{\prime}$, and $\boldsymbol{w}_{k}^{\prime} \in \mathbb{C}^{N \times 1}$ are the received signal, the effective channel matrix from the $i^{\prime}$-th user to the $k^{\prime}$-th user, the effective channel matrix from the $i$-th user to the $k^{\prime}$-th user, the effective noise, and the additive noise, respectively.

4) Detection Filter: In this paper, we use a linear filtering such as equalizations before decoding desired data. Let $\boldsymbol{U}_{k} \in$ $\mathbb{C}^{N \times D_{k}^{\prime}}$ and $\boldsymbol{U}_{k}^{\prime} \in \mathbb{C}^{N \times D_{k}}$ be the detection filter, such as the minimum mean-square error (MMSE), at the $k$-th user and at its partner, respectively. The $k$-th terminal user obtains a filtered signal $\tilde{\boldsymbol{x}}_{k}^{\prime}$ for decoding desired signal $\boldsymbol{x}_{k}^{\prime}$ with the filter $\boldsymbol{U}_{k}$, as follows:

$$
\begin{aligned}
\tilde{\boldsymbol{x}}_{k}^{\prime} & =\underbrace{\boldsymbol{U}_{k}^{\dagger} \boldsymbol{r}_{k}}_{\text {own }} \\
& =\underbrace{\boldsymbol{U}_{k}^{\dagger} \mathcal{G}_{k, k} \boldsymbol{V}_{k} \boldsymbol{x}_{k}}_{\text {desired }}+\underbrace{\boldsymbol{U}_{k}^{\dagger} \boldsymbol{\mathcal { H }}_{k, k} \boldsymbol{V}_{k}^{\prime} \boldsymbol{x}_{k}^{\prime}}_{\text {interference }} \\
& +\underbrace{\sum_{i \in \mathbb{N}_{K} \backslash k}\left(\boldsymbol{U}_{k}^{\dagger} \mathcal{G}_{k, i} \boldsymbol{V}_{i} \boldsymbol{x}_{i}+\boldsymbol{U}_{k}^{\dagger} \boldsymbol{\mathcal { H }}_{k, i} \boldsymbol{V}_{i}^{\prime} \boldsymbol{x}_{i}^{\prime}\right)}_{\text {noise }}+\underbrace{\boldsymbol{U}_{k}^{\dagger} \boldsymbol{\xi}_{k}} .
\end{aligned}
$$


Since the first term corresponds to the known transmitted signal, it can be readily cancelled out. The total number of interfering signals in the third term becomes $2(K-1)$. Both the detection filter $\boldsymbol{U}_{k}$ and the precoding filter $\boldsymbol{V}_{k}$ play an important role to suppress the last two terms.

5) Achievable Rate: The maximum data-rate $R_{k}^{\prime}$ at the $k$ th terminal user is given by the mutual information $\mathcal{I}\left(\tilde{\boldsymbol{x}}_{k}^{\prime} ; \boldsymbol{x}_{k}^{\prime}\right)$ for Gaussian signals as follows:

$$
R_{k}^{\prime} \leq \mathcal{I}\left(\tilde{\boldsymbol{x}}_{k}^{\prime} ; \boldsymbol{x}_{k}^{\prime}\right)=\log \frac{\left|\boldsymbol{U}_{k}^{\dagger} \boldsymbol{\Psi}_{k} \boldsymbol{U}_{k}\right|}{\left|\boldsymbol{U}_{k}^{\dagger} \boldsymbol{\Phi}_{k} \boldsymbol{U}_{k}\right|}
$$

where

$$
\begin{aligned}
& \boldsymbol{\Phi}_{k} \triangleq \sum_{i \in \mathbb{N}_{K} \backslash k}\left(\mathcal{G}_{k, i} \boldsymbol{V}_{i} \boldsymbol{V}_{i}^{\dagger} \mathcal{G}_{k, i}^{\dagger}+\boldsymbol{H}_{k, i} \boldsymbol{V}_{i}^{\prime} \boldsymbol{V}_{i}^{\dagger \dagger} \boldsymbol{\mathcal { H }}_{k, i}^{\dagger}\right)+\boldsymbol{\Sigma}_{k}, \\
& \boldsymbol{\Psi}_{k} \triangleq \boldsymbol{\Phi}_{k}+\boldsymbol{H}_{k, k} \boldsymbol{V}_{k}^{\prime} \boldsymbol{V}_{k}^{\prime \dagger} \mathcal{H}_{k, k}^{\dagger}, \\
& \boldsymbol{\Sigma}_{k} \triangleq \sigma^{2}\left(\boldsymbol{I}_{N_{k}}+\sum_{j \in \mathbb{N}_{K}} \boldsymbol{H}_{j, k}^{\dagger} \boldsymbol{P}_{j} \boldsymbol{P}_{j}^{\dagger} \boldsymbol{H}_{j, k}^{*}\right) .
\end{aligned}
$$

Note that any full-rank matrix $\boldsymbol{U}_{k}$ offers the identical rate. (For distributed interference alignment, the performance depends on $\boldsymbol{U}_{k}$ because it is used as a precoding filter as well.)

\section{Interference Alignment}

1) Basics of Zero-Leakage Interference Alignment: We briefly review an interference alignment technique [4], which avoids possible interference in a signal subspace at unintended receivers. Interference-free environment can be achieved by a precoding filter which casts the undesired signals into the null spaces of the signals as follows:

$$
\boldsymbol{U}_{k}^{\dagger} \mathcal{G}_{k, i} \boldsymbol{V}_{i}=\boldsymbol{U}_{k}^{\dagger} \boldsymbol{\mathcal { H }}_{k, i} \boldsymbol{V}_{i}^{\prime}=\mathbf{0},
$$

for any $i \neq k$. This interference alignment is opportunistically obtained in an iterative manner as in [7] when it is feasible.

Although such a nulling precoder achieves interferencefree environments (as if there are no interfering nodes) at each receivers, it is not optimal in the sense of sum-rate performance because it consumes the degrees of freedom only to suppress the interference not to enhance the desired signals. The precoding strategy to maximize signal-to-interferenceplus-noise power ratio (SINR) [7] or to minimize weighted mean-square error [8] is more relevant to achieve higher rate.

Our model of $K$-pair bidirectional relaying channels is different from $K$-user interference channels studied in [6]. The chief differences are three-fold: i) any terminal user plays a role as a source and a destination at the same time, ii) the number of interference signals becomes $2(K-1)$, and iii) the effective channel $\mathcal{H}_{k, i}$ or $\mathcal{G}_{k, i}$ is dependent on the precoders $\boldsymbol{V}_{i}$ and $\boldsymbol{V}_{i}^{\prime}$ due to the power constraint of $\boldsymbol{P}_{j}$.

2) Closed-Form Solution: One closed-form solution of a zero-leakage interference alignment is readily obtained for 2pair bidirectional relaying networks, given the effective channels $\boldsymbol{\mathcal { H }}_{k, i}$ and $\mathcal{G}_{k, i}$ (if we suppose that $\boldsymbol{P}_{j}$ is independent of $\left.\boldsymbol{V}_{i}\right)$. For simplicity, let us consider single stream transmission, $D_{i}=D_{i}^{\prime}=1$. For that case, precoding filters $\boldsymbol{V}_{i}$ and $\boldsymbol{V}_{i}^{\prime}$ become vectors, $\boldsymbol{v}_{i}$ and $\boldsymbol{v}_{i}^{\prime}$. We should align the interference from the user 1 and its partner to the same direction at the user 2 and its partner as follows:

$$
\frac{\mathcal{G}_{2,1} \boldsymbol{v}_{1}}{\left\|\mathcal{G}_{2,1} \boldsymbol{v}_{1}\right\|}=\frac{\mathcal{H}_{2,1} \boldsymbol{v}_{1}^{\prime}}{\left\|\mathcal{H}_{2,1} \boldsymbol{v}_{1}^{\prime}\right\|}, \quad \frac{\mathcal{H}_{2,1}^{\prime} \boldsymbol{v}_{1}}{\left\|\mathcal{H}_{2,1}^{\prime} \boldsymbol{v}_{1}\right\|}=\frac{\mathcal{G}_{2,1}^{\prime} \boldsymbol{v}_{1}^{\prime}}{\left\|\mathcal{G}_{2,1}^{\prime} \boldsymbol{v}_{1}^{\prime}\right\|} .
$$

It implies that the precoding vector should be an eigenvector of the channels:

$$
\boldsymbol{v}_{1}=\sqrt{\frac{E}{N}} \mathcal{V}\left(\mathcal{G}_{2,1}^{-1} \mathcal{H}_{2,1} \mathcal{G}_{2,1}^{\prime-1} \mathcal{H}_{2,1}^{\prime}\right),
$$

where $\mathcal{V}(\boldsymbol{X})$ denotes an eigen-vector of a matrix $\boldsymbol{X}$. In an analogous way, aligning interference at user 1 from user 2 , we obtain

$$
\boldsymbol{v}_{2}=\sqrt{\frac{E}{N}} \mathcal{V}\left(\mathcal{G}_{1,2}^{-1} \mathcal{H}_{1,2} \mathcal{G}_{1,2}^{\prime-1} \mathcal{H}_{1,2}^{\prime}\right) .
$$

Note that there are some degrees of freedom to choose which eigen-vector for data transmissions.

\section{Multiuser Detection (MUD)}

Although the capacity region of interference channels has been an open problem for decades, the achievable region derived by Han and Kobayashi is known as a good capacity bound [3]. The underlying idea is to split data into public and private information by superposition coding. The receivers decode successively all the public data and a private information for the intended users. This technique basically requires MUD which employs successive interference cancellations.

In this paper, we evaluate the advantage of MUD used in conjunction with interference alignment. For such a scenario, we can exploit the degrees of freedom to further maximize sum-rate performance because interference leakage can be treated by MUD. For instance, we can increase an undesired interference so that the receiver can perfectly decode it for interference cancellations. It achieves the interference-free channels even without zero-leakage interference alignment. To do so, we introduce hyper-layered superposition coding which multiplexes a larger number of low-rate codes than the maximum degrees of freedom, i.e., $D_{k} \gg N_{k}$, so that all the user can successively decode them for cancellations.

\section{Distributed Interference Alignment}

We use an iterative algorithm for distributed interference alignment proposed in [7], where each user requires only the local CSI. The channel reciprocity enables each user to optimize the precoding filter and the detection filter in an iterative manner through two-way links (forward and reverse links). The distributed method is summarized as follows:

- For forward links, each receiver uses the best detection filter $\boldsymbol{U}_{k}$ which suppresses interference based on some criteria described in the following section.

- For reverse links, each transmitter uses $\boldsymbol{U}_{k}$ as a new precoder $\boldsymbol{V}_{k}$ for transmissions.

This iterative procedure between forward and reverse links continues until the filters converge. Along this line of research, we discuss the advantage of MUD receivers which can cancel 
the strong interference leakage. Note that the distributed interference alignment is suited for bidirectional relaying networks since two-way links are used all along.

\section{A. Weak-Leakage Interference Alignment without MUD}

We describe several criteria in distributed interference alignment for single-user detection receivers. The precoding filter and the detection filter shall be designed to minimize interference level at unintended receivers. In the following, we present three criteria: min-leakage, MMSE, and max-SINR for the distributed interference alignment. Those criteria require only the local CSI: more specifically, the $k$-th receiver knows the effective channel matrix from the intended transmitter, $\mathcal{H}_{k, k} \boldsymbol{V}_{k}^{\prime}$, and the interference-plus-noise covariance $\boldsymbol{\Phi}_{k}$.

1) Min-Leakage Criterion: Total leakage of interference at the $k$-th receiver is written as

$$
\eta_{k}=\operatorname{tr}\left[\boldsymbol{U}_{k}^{\dagger}\left(\boldsymbol{\Phi}_{k}-\boldsymbol{\Sigma}_{k}\right) \boldsymbol{U}_{k}\right] .
$$

As derived in [7], the matrix which minimizes the leakage is obtained by the smallest $D_{k}^{\prime}$ eigenvectors of the interference covariance matrix, $\boldsymbol{\Phi}_{k}-\sigma^{2} \boldsymbol{I}_{N_{k}}$, as follows:

$$
\boldsymbol{U}_{k}=\mathcal{V}_{D_{k}^{\prime}}\left[\boldsymbol{\Phi}_{k}-\boldsymbol{\Sigma}_{k}\right] \boldsymbol{A}_{k},
$$

where $\mathcal{V}_{D}[\boldsymbol{X}]$ denotes the orthonormal matrix which contains the smallest $D$ eigenvectors of a matrix $\boldsymbol{X}$. We may introduce a diagonal matrix $\boldsymbol{A}_{k} \in \mathbb{C}^{D_{k}^{\prime} \times D_{k}^{\prime}}$ which controls the reception power of each stream.

2) MMSE Criterion: The MMSE filter is written as

$$
\boldsymbol{U}_{k}=\boldsymbol{\Psi}_{k}^{-1} \mathcal{H}_{k, k} \boldsymbol{V}_{k}^{\prime} \boldsymbol{A}_{k} .
$$

It minimizes mean-square error (MSE) between the weighted signal and the desired signal, namely $\mathbb{E}\left[\| \boldsymbol{U}_{k}^{\dagger}\left(\boldsymbol{r}_{k}-\right.\right.$ $\left.\left.\mathcal{G}_{k, k} \boldsymbol{V}_{k} \boldsymbol{x}_{k}\right)-\boldsymbol{A}_{k}^{\dagger} \boldsymbol{x}_{k}^{\prime} \|^{2}\right]$. Since the MMSE criterion considers the noise as well as interference, it generally outperforms the min-leakage criterion.

3) Max-SINR Criterion: As in [7], the SINR of signals for the $k$-th receiver and the $d$-th stream is written as

$$
\rho_{k, d}=\frac{\left|\boldsymbol{e}_{d}^{\dagger} \boldsymbol{U}_{k}^{\dagger} \boldsymbol{H}_{k, k} \boldsymbol{V}_{k}^{\prime} \boldsymbol{e}_{d}\right|^{2}}{\boldsymbol{e}_{d}^{\dagger} \boldsymbol{U}_{k}^{\dagger} \boldsymbol{\Phi}_{k} \boldsymbol{U}_{k} \boldsymbol{e}_{d}} .
$$

The detection matrix which maximizes SINR is obtained as

$$
\boldsymbol{U}_{k}=\boldsymbol{\Phi}_{k}^{-1} \mathcal{H}_{k, k} \boldsymbol{V}_{k}^{\prime} \boldsymbol{A}_{k} .
$$

The max-SINR criterion outperforms the min-leakage criterion and is comparable to the MMSE criterion in general.

\section{B. Strong-Leakage Interference Alignment with MUD}

Here, we address the case where the receiver employs successive interference cancellations for MUD. Since the MUD receivers can cancel strong interference, the precoding filter can exploit more degrees of freedom to enhance the desired signal power. We introduce a superposition coding to improve achievable rate of such strong-leakage interference channels.
1) Superposition Coding for MUD: The superposition coding is known as a good way to approach capacity region for multiple-access channels as well as interference channels, without using time sharing. The proposed superposition coding multiplexes a much larger number of low-rate data streams than the maximum available degrees of freedom, i.e., $D_{i} \gg N$. Let $x_{i}^{[d]}$ be the $d$-th stream transmitting from the $i$-th user, and $\boldsymbol{v}_{i}^{[d]}$ be the corresponding precoder vector. This data stream will be decoded by a set of users, $\mathbb{S}_{i}^{[d]} \subset \mathbb{N}_{2 K}$.

2) Distributed Interference Alignment with MUD: Suppose that the $k$-th receiver decodes $S_{k}$ interference signals $\left(x_{k_{1}}^{\left[d_{1}\right]}, x_{k_{2}}^{\left[d_{2}\right]}, \ldots, x_{k_{S_{k}}}^{\left[d_{S_{k}}\right]}\right.$ in the order $k_{s} \in \mathbb{N}_{K} \backslash\{k\}$ for $s \in \mathbb{N}_{S_{k}}$ ) before decoding all the desired signal $x_{k}^{\prime[d]}$. At the $s$-th stage, the receiver uses the detection vector $\boldsymbol{u}_{k}^{[s]}$ to decode $x_{k_{s}}^{\left[d_{s}\right]}$. If the actual rate of data $\boldsymbol{x}_{k_{s}}$ is set to be less than the maximum achievable rate at the $k$-th user of

$$
R_{k_{s}}^{\prime[k]}=\log \frac{\left|\boldsymbol{u}_{k}^{[s] \dagger} \boldsymbol{\Psi}_{k}^{[s]} \boldsymbol{u}_{k}^{[s]}\right|}{\left|\boldsymbol{u}_{k}^{[s] \dagger} \boldsymbol{\Phi}_{k}^{[s]} \boldsymbol{u}_{k}^{[s]}\right|},
$$

the receiver can completely cancel the interference. Here, we define

$$
\begin{aligned}
\boldsymbol{\Psi}_{k}^{[s]} & =\boldsymbol{\Psi}_{k}^{[s-1]}-\mathcal{H}_{k, k_{s-1}} \boldsymbol{v}_{k_{s-1}}^{\left[d_{s-1}\right]} \boldsymbol{v}_{k_{s-1}}^{\left[d_{s-1}\right] \dagger} \mathcal{H}_{k, k_{s-1}}^{\dagger}, \\
\boldsymbol{\Phi}_{k}^{[s]} & =\boldsymbol{\Psi}_{k}^{[s]}-\boldsymbol{\mathcal { H }}_{k, k_{s}} \boldsymbol{v}_{k_{s}}^{\left[d_{s}\right]} \boldsymbol{v}_{k_{s}}^{\left[d_{s}\right] \dagger} \boldsymbol{H}_{k, k_{s}}^{\dagger},
\end{aligned}
$$

At the last stage, the maximum achievable rate of the desired signal $\boldsymbol{x}_{k}$ can be improved up to $R_{k}^{\prime[k]}$.

In this paper, we focus on a greedy ordered successive cancellation which first tries to decode the maximum achievable rate (i.e., $\max _{i} R_{i}^{[k]}$ at the $s$-th stage) for a practical use. The iterative algorithm for distributed interference can be done by using the detection filter used at the last stage. In practice, the decodability can be decided by error-checking codes. Note that the receivers require additional CSIs for MUD.

We propose an iterative method for the distributed interference alignment which allows strong interference to be cancelled by MUD. It is summarized below.

1: Randomly generate an orthogonal precoder matrix $\boldsymbol{V}_{i}$ for each user $i \in \mathbb{N}_{K}$, such that $\operatorname{tr}\left[\boldsymbol{V}_{i} \boldsymbol{V}_{i}^{\dagger}\right]=E$. $D_{k}$ data of a rate $R_{k}$ are transmitted.

2: At the forward links, the $j$-th relaying node broadcasts the received signal $\boldsymbol{y}_{j}$ with a forwarding filter $\boldsymbol{P}_{j}$.

3: The $k$-th receiver computes the received covariance $\boldsymbol{\Psi}_{k}$ as in (8).

4: The greedy ordered successive cancellation based on $R_{k_{s}}^{k}$ is employed to decode $x_{k_{1}}^{d_{1}}, \ldots, x_{k_{S_{k}}}^{d_{S_{k}}}$, and $x_{k}^{\left[d_{k}\right]}$. The detection filter $\boldsymbol{U}_{k}^{\left[S_{k}+1\right]}$ at the last stage is used as a precoding matrix $\boldsymbol{V}_{k}$ for the reverse links.

5: At the reverse links, the $k$-th user computes the covariance $\boldsymbol{\Psi}_{k}$.

6: Update the achievable rate such that $R_{k}=\min _{k_{s}} R_{k}^{\left[k_{s}\right]}$.

7: Generate best weighting matrix $\boldsymbol{U}_{k}$ based on a filtering criterion to decode the intended data with a consideration of decodable interference. 


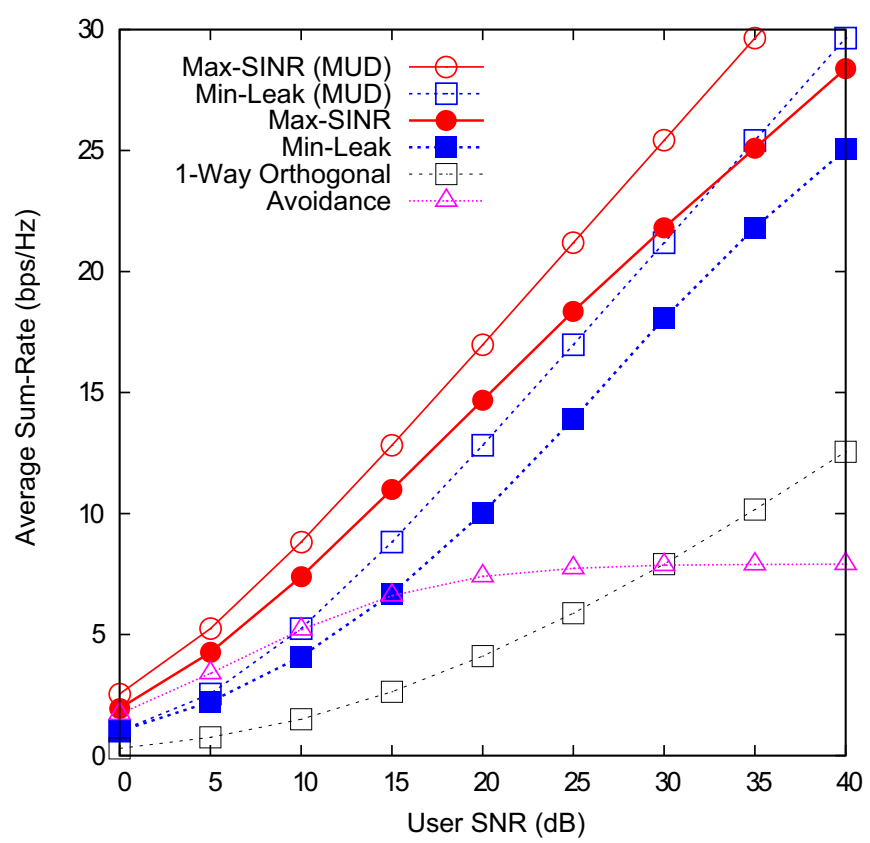

Fig. 2. Average sum-rate performance of distributed interference alignment as a function of user SNR for MUD receivers $(K=N=3)$.

8: Use $\boldsymbol{U}_{k}$ as a new precoder matrix $\boldsymbol{V}_{k}$ for the forward links.

9: Repeat from step 2 until convergence.

\section{Performance Evaluations}

We consider $K=3$ communicating pairs, each transmitter and receiver of which uses $N=3$ antenna branches. We assume that the channel is a frequency-flat block Rayleigh fading. We set the multiplexing degree $D_{k}=1$ for singleuser detection receivers. This parameter setting enables the iterative algorithm based on the min-leakage criterion to converge to zero-leakage interference with a high probability. For simplicity, we set a unit transmission power, $E=1$, and an identical channel gain, $\mathbb{E}\left[\left\|\boldsymbol{H}_{j, i}\right\|^{2}\right]=\mathbb{E}\left[\left\|\boldsymbol{G}_{j, i}\right\|^{2}\right]=$ $\mathbb{E}\left[\left\|\boldsymbol{H}_{j, i}^{\prime}\right\|^{2}\right]=\mathbb{E}\left[\left\|\boldsymbol{G}_{j, i}^{\prime}\right\|^{2}\right]=N$ for any $i, j \in \mathbb{N}_{K}$. The user SNR is then defined as $1 / \sigma^{2}$. We focus on the amplify-andforward scheme for the physical-layer network coding, such that $\boldsymbol{P}_{j}=\alpha_{j} \boldsymbol{I}_{N}$ with $\alpha_{j}$ being the amplification factor. It is assumed that each receiver has a perfect knowledge of CSI.

In Fig. 2, we show the averaged sum-rate performance of min-leakage and max-SINR criteria, as a function of user SNR. As references, we also present the performance curves achieved by an orthogonal scheme (in which each user communicates with the intended user assuming an equal timedivision multiple access with $2 K$ times power and eigenmode beamforming of $D_{k}=3$ multiplexing in one-way relaying) and the interference avoidance (which is a selfish approach proposed in [12]). One can see that distributed interference alignment offers an excellent performance compared to the other schemes. It is shown that the max-SINR criterion can considerably improve the sum-rate performance compared to the min-leakage criterion.

In Fig. 2, we also present the sum-rate performance for the case when MUD is used. We use $D_{k}=50$ for superposition coding. As shown in this figure, the strong-leakage interference alignment offers a performance improvement because of the signal cancellation by MUD receivers. More importantly, the sloop of the performance curves becomes slightly steeper. It implies that the allowance of strong interference at unintended users gives the filtering design more degrees of freedom to control signals.

\section{CONCLUSION}

In this paper, we investigated a distributed algorithm for interference alignment in $K$-pair bidirectional relaying systems which use analog network coding at the physical layer. It was shown that the distributed interference alignment works well as compared to the other existing methods. Furthermore, we proposed a joint interference alignment and MUD scheme, in which we superpose a larger number of low-rate coding than the maximum degrees of freedom. In this scheme, the transmitter only aligns partial interference while the receiver recovers the desired signal by suppressing strong interference via MUD. Through computer simulations, we demonstrated that the proposed interference alignment considerably improves sumrate performance as the MUD receivers can completely cancel strong interference.

\section{REFERENCES}

[1] T. M. Cover and J. A. Thomas, Elements of Information Theory, Second Edition, John Wiley \& Sons, 2006.

[2] A. B. Carleial, "A case where interference does not reduce capacity," IEEE Trans. IT, vol. IT-21, pp. 569-570, Sept. 1975.

[3] T. S. Han and K. Kobayashi, "A new achievable rate region for the interference channel," IEEE Trans. IT, vol. IT-27, pp. 49-60, Jan. 1981.

[4] S. Jafar and S. Shamai, "Degrees of freedom region for the MIMO X channel," IEEE Trans. IT, vol. 54, no. 1, pp. 151-170, Jan. 2008.

[5] V. R. Cadambe and S. A. Jafar, "Can feedback, cooperation, relays and full duplex operation increase the degrees of freedom of wireless networks?," Jan. 2007.

[6] V. R. Cadambe and S. A. Jafar, "Interference alignment and degrees of freedom of the $K$-user interference channel," IEEE Trans. IT, vol. 54, no. 8, pp. 3425-3441, Aug. 2008.

[7] K. Gomadam, V. R. Cadambe, and S. A. Jafar, "Approaching the capacity of wireless networks through distributed interference alignment," IEEE GLOBECOM, New Orleans, Nov.-Dec. 2008.

[8] D. A. Schmidt, C. Shi, R. A. Berry, M. L. Honig, and W. Utschick, "Minimum mean squared error interference alignment," Asilomar Conf. Signals, Systems, Computers, 2009.

[9] R. Tresch and M. Guillaud, "Cellular interference alignment with imperfect channel knowledge," IEEE ICC, June 2009.

[10] M. Maddah-Ali, A. Motahari, and A. Khandani, "Signaling over MIMO multi-base systems: Combination of multi-access and broadcast schemes," IEEE ISIT, Seattle, USA, July 2006,

[11] N. Lee, J.-B. Lim, and J. Chun, "Degrees of freedom of the MIMO Y channel: Signal space alignment for network coding," IEEE Trans. IT, vol. 56, no. 7, July 2010.

[12] C. Rose, S. Ulukus, and R. Yates, "Wireless systems and interference avoidance," IEEE Trans. Wireless Commun., vol. 1, pp. 415-428, July 2002. 